\title{
Alcohol as a cue for extinction: State dependency produced by conditioned inhibition
}

\author{
CHRISTOPHER L. CUNNINGHAM \\ University of Oregon Health Sciences Center, Portland, Oregon 97201
}

\begin{abstract}
A conditioned-emotional-response (CER) paradigm was used in two experiments to evaluate the effects of alcohol on extinction. In both experiments, rats received tone-shock pairings without alcohol and then received extinction trials either with or without alcohol (injections of saline, .75 or $1.5 \mathrm{~g} / \mathrm{kg}$ ethanol). The high dose of alcohol suppressed baseline barpressing for food reward, but there was only weak evidence (Experiment 2) that it enhanced responding during the tone early in extinction. In both experiments, extinction of CER under the high alcohol dose was found to be state dependent, that is, postextinction tests after saline injection showed a reinstatement of suppression to the tone. Experiment 2 indicated that this effect could be attributed to alcohol's becoming a conditioned inhibitory stimulus as a result of its association with extinction. This supports the general suggestion that situational stimuli normally become inhibitory during the course of simple extinction and may have implications for the role that state-dependent learning plays in drug dependence.
\end{abstract}

Alcohol has been found to retard extinction of instrumental locomotor behavior based both on appetitive (Barry, Wagner, \& Miller, 1962) and on aversive reinforcement (Amit, Ziskind, \& Baum, 1973; Baum, 1969, 1970, 1971; Skurdal, Eckardt, \& Brown, 1975). Barry et al. attributed their results to the drug's selective ability to reduce inhibition caused by nonreinforcement-specifically, by decreasing the intensity of anticipatory frustration. Moreover, because performance on later extinction trials did not depend on the presence or absence of alcohol on early extinction trials, they concluded that the drug's effect was primarily on the performance and not the learning of inhibition.

The analysis by Barry et al. specifically excluded the possibility that alcohol could exert a similar effect on the extinction of an aversively motivated response, presumably because such extinction did not involve anticipatory frustration. Nevertheless, the studies of Baum and his colleagues on shock-avoidance responding and that of Skurdal et al. on shockescape responding provide convincing evidence that alcohol maintains performance during the extinction of aversively motivated behavior. Although Baum (1969) originally suggested that his data were consistent with the general statement that alcohol enhances performance by weakening response-inhibiting tendencies, he has also offered two other, almost contradictory, explanations of alcohol's performance-

This research was supported by a departmental training grant (AA07074) and by a research grant to Judson S. Brown (AA01229) from the National Institute on Alcohol Abuse and Alcoholism. Reprint requests should be sent to the author at the Department of Medical Psychology, University of Oregon Health Sciences Center, Portland, Oregon 97201 , enhancing effects. The first of these relies on the possibility that the function relating avoidance performance and arousal is nonmonotonic (i.e., an inverted $U$ ) and that alcohol may actually improve performance by decreasing arousal (Baum, 1969). According to the second explanation, the drug state is viewed as being aversive (for drug-naive organisms) and, by summating with fear in the avoidance situation, is said to increase resistance to extinction (Amit \& Baum, 1970; Amit et al., 1973; Baum, 1971). It should be noted that, by making a special assumption about the performance-arousal function, the former explanation remains consistent with traditional beliefs about alcohol's tension-reducing abilities, whereas the latter explanation asserts that alcohol (at least when it is novel) has the opposite effect (cf. Cappell \& Herman, 1972).

Although all of the aforementioned studies are consistent with the belief that alcohol affects extinctive inhibition, it seems possible that its performance-enhancing effects are more due to the use of a locomotor response measure than to any general effect of alcohol on the extinction process. That is, these studies may simply reflect alcohol's apparent tendency to increase general motor activity at low dose levels (e.g., Matchett \& Erickson, 1977). Two recent aversive-conditioning experiments support this view by showing that alcohol enhances locomotor behavior even in the presence of a stimulus which has never been associated with shock (DiGiusto \& Bond, 1977; Cunningham, Note 1). This view seems to be supported further by the fact that Barry et al.'s subjects showed enhanced running under alcohol from the onset of extinction, before anticipatory frustration could presumably have become an important determinant of performance. 
The present experiments were undertaken to provide a further assessment of alcohol's impact on extinction. They involved use of the conditionedemotional-response (CER) paradigm, which differs in at least two potentially important ways from those described above. First, the target behavior does not involve running, and second, the acquisition and extinction of the conditioned response can be viewed primarily in terms of Pavlovian contingencies and not as a combination of Pavlovian and instrumental contingencies. With respect to this latter distinction, it is possible that alcohol's effect is restricted to inhibition caused by nonreinforcement of a response established by instrumental contingencies.

\section{EXPERIMENT 1}

This study was intended to provide dose-response information on alcohol's effects during CER extinction. Three groups of rats were first trained to barpress for food reward on a variable-interval (VI) schedule, and then, after placebo injections, given Pavlovian conditioning trials while barpressing. Before each subsequent extinction session, two groups received an injection of ethanol $(.75$ or $1.5 \mathrm{~g} / \mathrm{kg}$ ), and the third group received a placebo injection. To provide additional information on the nature of any changes observed during extinction, the drug conditions were reversed at the end of extinction.

\begin{abstract}
Method
Subjects

The subjects were 22 female hooded rats, about 10 months old at the beginning of VI training. Several months before this experiment, they had been food deprived and trained to barpress on a variable-ratio schedule for food reward. Prior to VI training in the present experiment, they were redeprived and gradually reduced to $80 \%$ of their ad-lib body weights. All rats were caged individually and water was available in the home cage at all times.
\end{abstract}

\section{Apparatus}

Four operant-conditioning chambers $(22.5 \times 23 \times 19 \mathrm{~cm}$, inside) were used. Each chamber had a foodcup centered at the bottom of one of the aluminum end panels and a lever attached to the wall at the left of the foodcup, $2.5 \mathrm{~cm}$ above the floor. The side walls and ceiling were made of clear acrylic, and the floor consisted of $2.3-\mathrm{mm}$ stainless steel rods mounted $1.27 \mathrm{~cm}$ apart. The grid floor was connected via a relay-sequence scrambler to a high-voltage $(480-\mathrm{V} \mathrm{ac})$ shock source through a series resistor $(820 \mathrm{k} \Omega)$. When activated, this system applied a current of about $.5 \mathrm{~mA}$ to a rat whose resistance was $150 \mathrm{k} \Omega$.

Each operant chamber was contained in a ventilated, lightand sound-attenuating enclosure $(36 \times 71 \times 34 \mathrm{~cm})$. A $6-\mathrm{W}$ houselight (normally off) was mounted on one end of this enclosure, and a speaker was located over the center of the operant chamber. This speaker permitted delivery of a $2000-\mathrm{Hz}$ tone at about $85 \mathrm{~dB}$ (re $20 \mu \mathrm{N} / \mathrm{m}^{2}$ ). A digital laboratory computer controlled experimental contingencies and recorded barpresses.

\section{Procedure}

During the first $30 \mathrm{~min}$ of the first session, all rats were reinforced for barpressing with food pellets (45 $\mathrm{mg}$, Noyes) on a VI 1-min schedule. This schedule was changed to VI $2 \mathrm{~min}$ during the second $30 \mathrm{~min}$. Subsequent sessions were also $1 \mathrm{~h}$ long, and training on the VI-2 schedule continued for an additional nine sessions.

During the next two sessions, all rats were exposed while barpressing to the $2,000-\mathrm{Hz}$ tone that would be used during subsequent conditioning. Two 2 -min presentations were given in each session, occurring approximately 20 and $40 \mathrm{~min}$ ( $\pm 5 \mathrm{~min}$ ) after its start. A total of five fear-conditioning sessions followed these pretest days. The fear-conditioning sessions were identical to the pretest sessions except that each tone presentation terminated with the onset of a .5-sec, .5-mA footshock. Two VI sessions without stimulus presentations intervened between the third and fourth fear-conditioning sessions in order to permit recovery of baseline (pre-CS) levels of VI responding, which had been depressed somewhat as a result of the conditioning procedure. All pretest and fear-conditioning sessions were preceded by IP injections of normal saline $(10 \mathrm{ml} / \mathrm{kg})$.

At the end of fear conditioning, one rat was eliminated for failing to show evidence of suppression to the tone [suppression ratios (see below) of $.46, .46$, and .47 , respectively, on the last 3 days of conditioning]. Three groups ( $n=7 /$ group) were formed from the remaining animals, matched for their performance on the last 2 conditioning days. All groups then received nonreinforced (extinction) presentations of the tone during five subsequent sessions. These sessions were identical to pretest sessions, except that each group received a different injection prior to each extinction session. Group .75 received a $.75-\mathrm{g} / \mathrm{kg}$ injection of ethanol in a normal saline solution $(9.5 \%, v / v)$. Group 1.5 received a $1.5-\mathrm{g} / \mathrm{kg}$ ethanol injection $(19 \%, v / v)$. Group Saline was injected with an equivalent volume of normal saline $(10 \mathrm{ml} / \mathrm{kg})$.

A sixth extinction session was given following a reversal in drug conditions. The Saline group was tested with the low ethanol dose and the two ethanol groups were tested following a saline injection. The seventh session was a return to the original extinction-drug conditions, and the eighth session, a second reversal. During this second reversal, the Saline group was tested with the high ethanol dose.

Both absolute response rates and a relative-rate-change measure (suppression ratio) are reported as dependent variables. The ratio was calculated by dividing the number of responses occurring during the $\mathrm{CS}$ by the total number of responses recorded during the CS and an equally long pre-CS (baseline) period. A ratio of .5 indicates that no change in rate was occasioned by the CS, whereas ratios above and below .5 reflect response facilitation and suppression, respectively.

\section{Results and Discussion Conditioning and Extinction}

The left side of Figure 1 depicts the average suppression ratios for each group over the last 2 fear conditioning days and on each of the 5 extinction days. Group 1.5's average ratio on the 1st day of extinction does not include scores from two rats which failed to respond during the 2-min pre-CS intervals on that day. Group differences were not significant either at the end of conditioning or on any of the extinction days. The loss in suppression between the 1st and 5th extinction days was reliable for each group (all Wilcoxon $\mathrm{Ts}<1, \mathrm{p}<.05$ ).

Baseline barpress rates during conditioning and extinction (not shown in the figure) were derived 


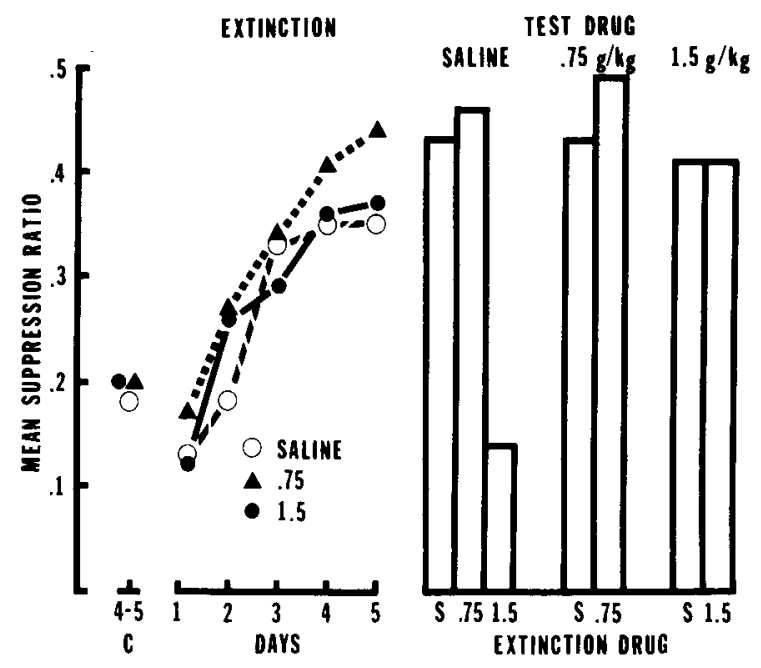

Figure 1. The left panel shows mean suppression ratios during tone trials on the final 2 days of conditioning $(C)$ and on each of the 5 extinction days. The right panel shows suppression during drug-reversal tone tests. Each set of bars represents a different test-drug condition. Each group's extinction-drug condition is labeled along the abscissa ( $S=$ normal saline; $.75=.75 \mathrm{~g} / \mathrm{kg}$ ethanol; $1.5=1.5 \mathrm{~g} / \mathrm{kg}$ ethanol).

from the number of responses recorded during the 2-min interval before each CS presentation. The mean baseline rates over the last 2 conditioning days were $5.7,8.2$, and 8.7 responses/min for Groups Saline, .75 , and 1.5 , respectively. On the 1 st day of extinction, those rates were $6.4,6.8$, and 3.2 , whereas on the 5 th day they were $8.5,7.1$, and 9.5 responses/min, respectively. Mann-Whitney U tests revealed no significant differences between groups on the final conditioning days or on any of the extinction days. Within-group comparisons indicated that Group 1.5's rate was depressed by the introduction of alcohol, but that it recovered over the course of extinction. Its average rate on the 1 st extinction day was reliably below its rate over the last 2 conditioning days $(T=1, p<.05)$ and below its rate on the 5 th extinction day $(T=0, \mathrm{p}<.05)$. Similar comparisons yielded no differences for the other two groups.

\section{Drug-Reversal Test}

The right side of Figure 1 shows the average suppression ratios under each test drug condition averaged over the two drug-reversal sessions (except for Group Saline) and the last two extinction-drug sessions (Days 5 and 7). As can be seen in the second and third sets of bars, the Saline group did not differ from the ethanol groups when tested under either ethanol dose level. However, during Saline tests (first set of bars), Group 1.5 was reliably more suppressed than Group Saline (Mann-Whitney U = 3 ) and Group $.75(U=2, p<.01)$. The latter groups did not differ during the saline tests.
Analysis of CS response rates supported conclusions derived from the suppression ratios about the effects of drug reversal. The average $\mathrm{CS}$ rates of the Saline, .75, and 1.5 groups during the saline tests were $8.5,5.9$, and 2.3 responses $/ \mathrm{min}$, respectively. Group 1.5's rate was significantly below those of Group Saline $(U=5)$ and Group .75 $(\mathrm{U}=6, \mathrm{p}<.02)$. Groups Saline and $.75 \mathrm{did}$ not differ. Appropriate group comparisons of CS rate under each ethanol dose level indicated no other differences. Baseline (pre-CS) response rates did not differ under any of the test-drug conditions.

In summary, although the extinction data suggest no effect of ethanol other than temporarily to depress base rate under the high dose, the drugreversal data show that CER extinction was specific to the drug condition in the $1.5 \mathrm{-g} / \mathrm{kg}$ group. It is interesting to note that the reinstatement of fear produced by the removal of alcohol was virtually complete in that group.

If one were to consider only the data from the final saline test, one might conclude that this experiment provides evidence of alcohol's extinctionretarding abilities. The behavior of Group 1.5 in that test could be construed as indicating that no extinction to the CS had occurred despite that group's having received the same number of nonreinforced exposures to the tone found sufficient to eliminate suppression in the control group. However, the facts that suppression persisted when alcohol was introduced in extinction and that it progressively declined over extinction trials suggest further that the drug-reversal effect was probably not due to any influence of alcohol on the development of extinctive inhibition, but was due instead to the association of extinction with the drug state. In other words, the outcome resembles an instances of "statedependent learning" (cf. Overton, 1972)-the loss in suppression to the tone observed in the alcohol state failed to transfer to the no-alcohol state.

\section{EXPERIMENT 2}

Because the groups in the first experiment differed in the amount of their previous exposure to alcohol at the time of the drug-reversal test, it is possible that the outcome of that test did not depend upon the presence of alcohol during extinction. Thus, Experiment 2 was designed to replicate that outcome and to confirm that it depended upon the correlation of alcohol with extinction. This was done by giving the control group equivalent exposure to alcohol during the extinction phase, but not during actual extinction sessions. Since the low dose proved ineffective in Experiment 1, only the high dose was used in the present experiment. 
Experiment 2 was also intended to illuminate the nature of the state-dependent learning supposed to have occurred in the first experiment. Specifically, the possibility that inhibition had accrued to alcohol during extinction (i.e., that alcohol had become a conditioned inhibitory stimulus) was assessed by examining alcohol's subsequent impact on suppression produced by a second, nonextinguished CS. Presumably, if alcohol's stimulus features had acquired inhibitory properties, one would expect to see less suppression to the second CS under alcohol in a group previously extinguished under alcohol than in a group extinguished under saline (Rescorla, 1969).

\section{Method}

\section{Subjects and Apparatus}

The subjects were 28 naive, female albino rats about 135 days old at the beginning of VI training. Over a period of several weeks prior to VI training, they were gradually reduced to $80 \%$ of their initial ad-lib body weights by restricting daily food intake. All rats were caged individually, and water was available in the home cage at all times. The apparatus was that used in Experiment 1.

\section{Procedure}

All rats were magazine-trained with food pellets delivered on a variable-time 1-min schedule in the first session. In addition, each barpress produced a food pellet. This training continued until each rat had made about 50 barpresses. Subsequent sessions were $1 \mathrm{~h}$ long and involved reinforcement on VI-1 and VI-2-min schedules, as in the first experiment.

A single pretest session occurred on the day after the 10th VI-training session. Two 2 -min presentations each of a $2,000-\mathrm{Hz}$ tone and a flashing houselight $(2 / \mathrm{sec})$ were given, at a mean intertrial interval of $10 \mathrm{~min}$. During each of the next eight $1-\mathrm{h}$ sessions, one light and one tone fear-conditioning trial were given. These trials occurred about 20 and $40 \mathrm{~min}( \pm 5 \mathrm{~min})$ after the start of the session and terminated with the presentation of a .5-sec, .5 -mA footshock. The pretest and fear-conditioning sessions were preceded by IP injections of normal saline $(10 \mathrm{ml} / \mathrm{kg})$.

Two groups were formed ( $n=14 /$ group), matched for their performance on the last 2 conditioning days, and their fear of the tone was extinguished on alternate days over the next 18 sessions. Each of the nine extinction sessions consisted of two nonreinforced presentations of the tone. No stimuli were presented during the VI sessions which intervened between extinction sessions. Group Alcohol received an injection of ethanol $(1.5 \mathrm{~g} /$ $\mathrm{kg}$ ) before each extinction session and an injection of saline before each VI session. These conditions were reversed for Group Saline. Thus, the groups were equated for barpressing experience under the drug and no-drug conditions and received the same number of nonreinforced tone trials. They differed, however, as to which drug condition prevailed during the extinction trials.

By the 9th extinction session, there was very little suppression during the tone in either group, and drug conditions were reversed during a 10 th extinction session. All rats were then returned to their original extinction-drug conditions for one more extinction session.

Tests for suppression to the light were given under the drug and no-drug conditions during the next two 1-h sessions. Two nonreinforced light trials occurred in each session. Half of the rats in each group were tested after a saline injection in the first session and after an ethanol injection in the second session. This order was reversed for the remaining animals.
These light tests indicated no differences between groups either in the saline condition (where both groups showed low suppression ratios) or in the alcohol condition (where neither group suppressed). The strong unconditioned fear-inhibiting effects of alcohol observed in the Saline Group were unexpected (see Discussion), but it seemed that those effects might have obscured any group differences in alcohol's conditioned inhibitory properties because of a "ceiling effect" (if alcohol's unconditioned properties were sufficient to inhibit all fear of the light, it would not be possible to measure the added contribution of conditioned inhibition). To evaluate this possibility, the light was reinforced with footshock on half of the trials in the presence of alcohol during three subsequent sessions. Presumably, such a procedure would gradually eliminate alcohol's fear-inhibiting properties, whether conditioned or unconditioned, and perhaps allow a better test of group differences at a mid-range level of suppression. Since both groups had an identical history of excitatory conditioning to the light, differences in suppression during alcohol-light conditioning could only reflect differences in the inhibitory value of alcohol.

\section{Conditioning}

\section{Results and Discussion}

The average suppression ratios for each group on the last 2 days of conditioning were .06 for the tone and .08 for the light. Barpress response baselines on those days averaged 16.2 and 14.7 responses/min for Groups Alcohol and Saline, respectively. There were no group differences on either measure.

\section{Extinction}

Response rates. The left panel of Figure 2 depicts mean baseline response rates both on extinction days and on intervening VI days. These data indicate a general suppression of barpressing under alcohol. Each animal's overall rate under alcohol was less than its overall rate under saline. Over days, both groups showed an increase in baseline responding. Within-groups comparisons of average rate between the first 4 days and last 4 days indicated that the increase was reliable under both drug conditions (all $\mathrm{Ts} \leqslant 18, \mathrm{p}<.05$ ). Further analysis revealed that the magnitude of the increase in baseline did not differ across drug conditions in either group ( $T s \geqslant 29)$.

The right-hand panel of Figure 2 shows mean response rates during the tone $C S$ in extinction sessions. Although Group Alcohol's CS response rate was slightly enhanced relative to that of Group Saline initially, Group Saline's CS rate eventually exceeded that of Group Alcohol. Between-group comparisons of $\mathrm{CS}$ rates indicated that differences were not significant on any of the first 7 extinction days. Within-group comparisons revealed that Group Alcohol's average CS rate across the first 2 extinction days reliably exceeded its average rate for the last 2 conditioning days $(T=10, N=11, p<.05)$, whereas that of Group Saline did not $(T=22.5$, $N=13)$. Analyses of the final 2 extinction days yielded significant group differences $(\mathrm{Us}=32$ and 39.5 , respectively, $\mathrm{p}<.02$ ). As can be seen by com- 

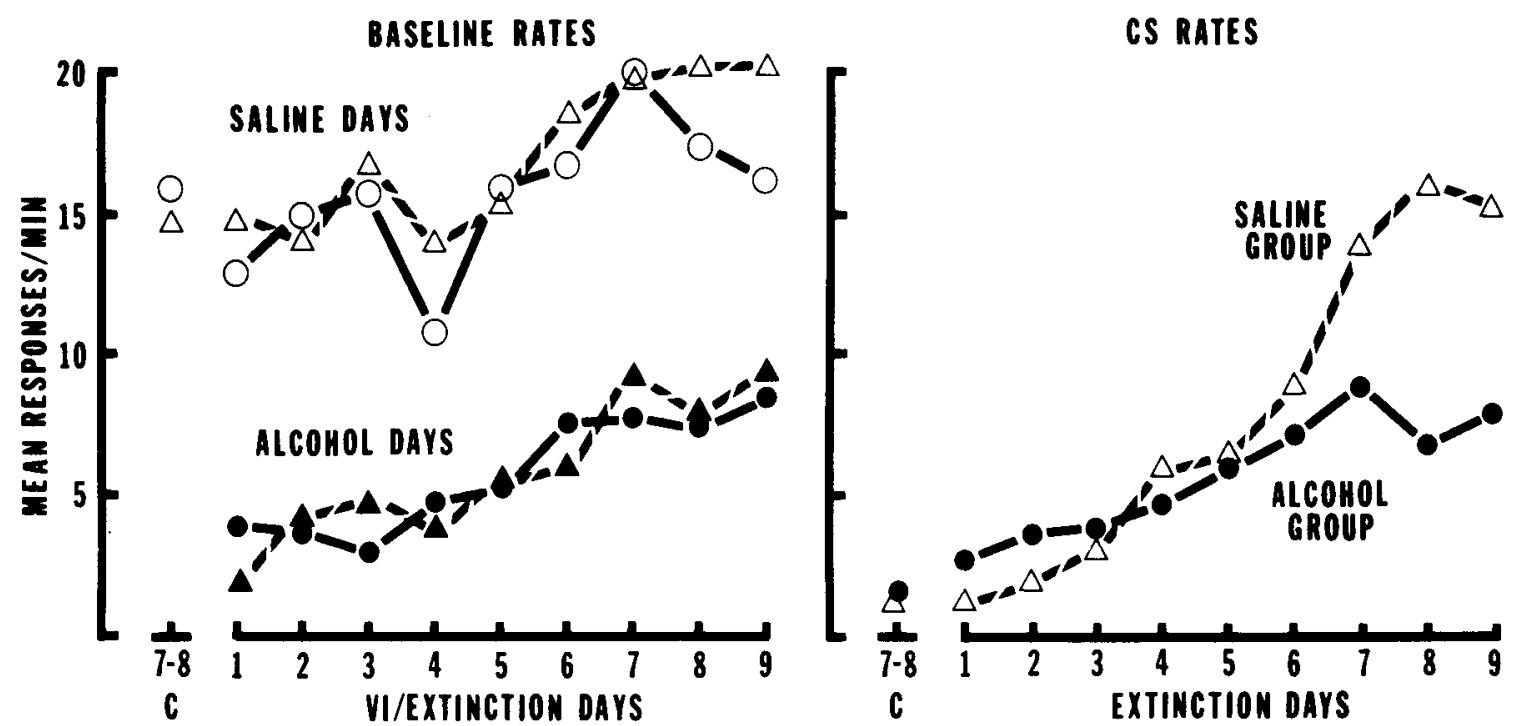

Figure 2. The left panel depicts mean baseline response rates during the 2-min pre-CS interval on the last 2 days of conditioning (C) and on each of the 9 extinction days. Also shown in the left panel are the baseline rates during the VI sessions which intervened between each extinction session. The right panel depicts mean response rates during the $2-$ min tone CS on the final conditioning days and on each extinction day. In each panel, circles represent the Alcohol group and triangles, the Saline group. Open symbols depict data obtained after saline injection, whereas closed symbols depict data obtained after alcohol injection.

paring extinction day response rates across the panels of Figure 2, each group's CS rate (right panel) gradually approached its baseline rate (left panel) over days.

Suppression ratios. Average daily suppression ratios were calcuated for each group based on the individual means of rats responding on at least one trial each day (ratios were not calculated unless one or more responses were recorded during the 2-min pre-CS interval). Group Saline's mean ratios on the $1 \mathrm{st}, 5$ th, and 9 th extinction days were $.08, .29$, and .45 , respectively. Group Alcohol yielded ratios of .31 , .47 , and .49 , respectively. Group differences were reliable on all but the last 2 days of extinction (al Us $<41$, ps $<.05$ ). Interpretation of comparisons on the initial extinction days is complicated, however, by the fact that, while Group Saline rats responded during all pre-CS intervals, many Group Alcohol rats did not. The percentage of trials on which ratios were calculated for Group Alcohol gradually increased from a low of $54 \%$ on Day 1 , to $89 \%$ on Day 5 , and finally to $100 \%$ on Day 9 .

\section{Drug-Reversal Test (Tone)}

Data from the drug-reversal test were compared to data from the extinction-drug sessions before and after the reversal test. The outcomes of these analyses were the same, regardless of which extinction day was compared to the drug-reversal day. For simplicity, the average scores from these extinction days were used in the analyses described below.

Suppression ratios. The left side of Figure 3 depicts suppression ratios during the drug-reversal test. When tested under alcohol (second set of bars), the groups did not differ and neither group showed appreciable suppression. However, when tested after saline injection (first set of bars), the Alcohol group was significantly more suppressed than the Saline group $(\mathrm{U}=15, \mathrm{p}<.01)$. Thus, as in Experiment 1 , the group whose fear was extinguished under the influence of alcohol showed a reinstatement of fear when subsequently tested in the absence of alcohol.

Response rates. The analysis of CS response rates during the drug-reversal test mirrored the analysis of suppression ratios. The CS rates during alcohol sessions were only slightly below the alcohol baseline rates and did not differ between groups (means of 7.8 and 9.8 responses/min for Groups Alcohol and Saline, respectively). Comparison of response rates during the tone after saline injection indicated that Group Alcohol's rate (5.0) was significantly below that of Group Saline (16.2) $(U=19.5, \mathrm{p}<.01)$.

Baseline response rates after alcohol injection were 8.4 and 10.6 responses/min for Groups Alcohol and Saline, respectively. After saline injection, baseline rates were 18.5 and 21.3 responses/min, respectively. The groups did not differ reliably under either drug condition, but each group's rate under alcohol was significantly less than its rate under saline (Ts = 11 and 2, ps $<.01$, for Groups Alcohol and Saline, respectively).

\section{Light Test}

As indicated earlier, no group differences in responding during the nonextinguished light stimulus were initially seen under either drug condition. The average suppression ratios on the light-test days are shown in the middle portion of Figure 3. Withingroup comparisons indicated significant differences between drug conditions both for suppression ratios 


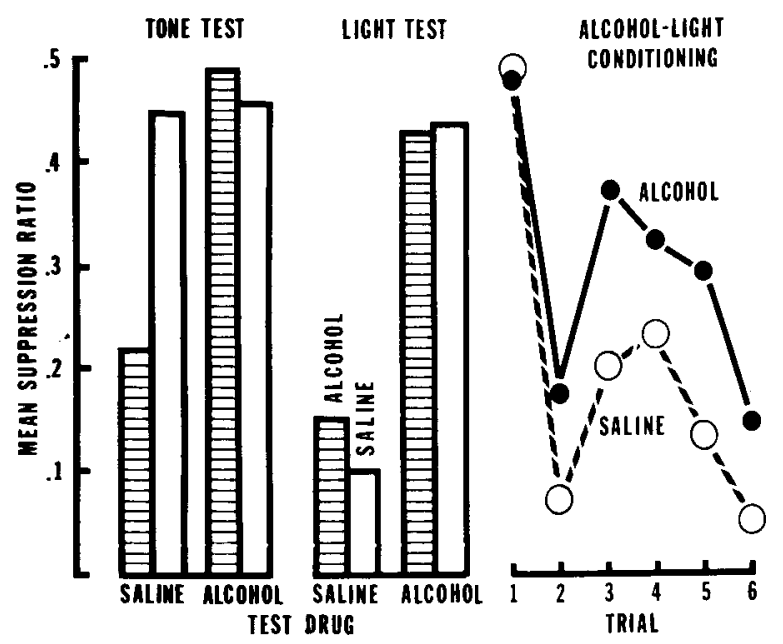

Figure 3. The left panel shows mean suppression ratios for the Alcohol group (cross-hatched bars) and Saline group (open bars) during postextinction, drug-reversal tone tests. The first set of bars shows performance after saline injection. And the second set shows performance after alcohol injection. The center panel similarly depicts suppression to the light stimulus for both groups during saline and alcohol tests. The final panel shows suppression to the light in the presence of alcohol on each trial during partial reinforcement conditioning.

(both $\mathrm{Ts}=0, \mathrm{p}<.01$ ), and for response rates during the light (Ts $=2$ and 5, ps $<.01$, for Groups Alcohol and Saline, respectively). Group Alcohol's mean CS rate during the saline test was 2.1 and Group Saline's was 1.7 responses/min. After alcohol injection, the respective rates were 8.1 and 7.7 responses/min. It should be noted that these increases in CS rate under alcohol occurred despite alcohol's depressant effect on pre-CS baseline rate (saline baselines $=18.1$ and 18.5, whereas alcohol baselines $=10.7$ and 12.2 responses/min for Groups Alcohol and Saline, respectively).

\section{Alcohol-Light Conditioning}

Alcohol's differential ability to inhibit fear in each group emerged when the light was partially reinforced with shock. The mean suppression ratios on each of the six trials in this phase are shown in the right side of Figure 3. As can be seen, after the first trial, Group Alcohol consistently showed less suppression during the light than did Group Saline. A comparison of the overall average suppression ratios (excluding the trial before the first shock) indicated that the group difference was reliable $(U=56, p<.05)$. Analysis of response rates during the CS also indicated a significant group difference. Group Alcohol's average CS rate on Trials 2-6 was 4.5, whereas that of Group Saline was $1.6 \mathrm{responses} / \mathrm{min}(U=54.5$, $\mathrm{p}<.05$ ). The baseline rates did not differ (Group Alcohol $=11.0 ; \quad$ Group Saline $=12.7$ responses/ $\min$ ).

In summary, Experiment 2 replicated the major outcome of the first experiment. After extinction under the influence of alcohol, suppression to the tone was reinstated by the removal of alcohol. Moreover, this experiment provides evidence that, as a result of its correlation with the extinction procedure, alcohol developed conditioned inhibitory properties. Experiment 2, however, differed in certain important aspects from the first experiment. First, in contrast to the weak, transient depression of baseline response rates seen in Experiment 1, the $1.5-\mathrm{g} / \mathrm{kg}$ dose appeared to exert a greater and longer-lasting decremental effect on them in the present experiment. This was likely due to differences in ethanol tolerance attributable either to rat strain (Deutsch \& Hardy, 1976) or to the longer recovery periods between ethanol exposures in the second experiment (cf. LeBlanc \& Cappell, 1975). A second, possibly related difference between the two experiments is in the performance observed during extinction under alcohol. The first experiment indicated no differential effect of alcohol on extinction performance, whereas the second suggested an alleviation of suppression during extinction. However, interpretation of the ratio measure must be tempered by consideration of absolute response rates (cf. Blackman, 1977). In this instance, the depression in baseline rates under alcohol would appear to account almost entirely for the elevation in the suppression-ratio measure. There was only a slight hint that alcohol actually increased rate of responding during the CS early in extinction.

Somewhat surprising is the finding that alcohol attenuated suppression to the light in the Saline group during the initial light test. The effect was rather substantial and appeared both in the CS-rate and suppression-ratio measures. It was unexpected for two reasons: First, as just mentioned, this apparently unconditioned inhibition did not appear to be as strong during tone extinction under alcohol in either experiment; second, previous studies of alcohol's impact on conditioned suppression have uniformly failed to find any influence of the drug on CS-evoked suppression (Cicala \& Hartley, 1967; Dickerson \& Ferraro, 1976; Goldman \& Docter, 1966; Lauener, 1963). Several features of the present situation may have promoted the occurrence of this effect. For instance, it may be that alcohol has different effects on stimuli in the visual and auditory modalities. A greater disruption by alcohol of responses conditioned to visual stimuli would account for the present results and might also explain previous failures. In all four of the studies cited above, the CS consisted of an auditory stimulus or contained an auditory component. Another aspect of the present experiment which may have been important is that the Saline group was more familiar with alcohol at the time of the light test than the Alcohol group had been at the start of tone extinction. A reduction in alcohol's novelty or the 
development of tolerance to its motor-impairing effects may have been necessary for revealing alcohol's unlearned inhibitory properties in this situation. However, this could not account for previous failures to see alleviation of CS-induced suppression where extensive exposure to ethanol has been given (e.g., Dickerson \& Ferraro, 1976). As an aside, it might be noted that the absence of shock during testing was probably critical in obtaining loss of suppression to the light under alcohol in the saline group. The right-hand portion of Figure 3 reveals that a single shock in the presence of alcohol was sufficient to produce a level of suppression to the light equivalent to that seen earlier during testing under saline.

\section{GENERAL DISCUSSION}

The most consistent and striking outcome of both experiments was not the effect of alcohol on performance during extinction, but the finding that extinction under alcohol failed to transfer to the noalcohol condition. This finding appears to fit general descriptions of "dissociated" or state-dependent learning (Overton, 1972). The outcome of the subsequent test of alcohol's effect on behavior in the presence of a nonextinguished CS (Experiment 2) suggests that the "dissociation" in this instance was not due to a drug-produced interference with extinction, but was due instead to the drug's cue properties. Moreover, that test showed that alcohol did not merely produce a "state" or "context" in which the animal learned to be unafraid of the tone. Rather, as a result of its association with the nonreinforcement of an established excitatory stimulus, alcohol acquired a more general ability to inhibit excitation based on shock reinforcement.

These experiments do not show the extinctionretarding effects of alcohol reported in instrumental learning situations (Amit et al., 1973; Barry et al., 1962; Baum, 1969, 1970, 1971; Skurdal et al., 1975). In fact, several of the results noted here would suggest that alcohol may actually enhance extinction of CER, although this interpretation is complicated by the drug's depressant effect on baseline responding. The difference between the present studies and those cited above may indicate that alcohol affects inhibition generated by nonreinforcement of a response established by instrumental contingencies but does not affect inhibition generated by Pavlovian extinction. Alternatively, as mentioned earlier, the effects observed in the instrumental learning studies may reflect their use of a locomotor response measure and not an effect of alcohol on extinctive inhibition.

The present results may have implications for two general issues: the role of inhibition in extinction and the role of state-dependent learning in drug dependence. With respect to the first of these issues, the finding that a "situational stimulus" (alcohol intoxication) acquires inhibitory properties during extinction complements Rescorla's (in press) recent report that the compounding of a discrete external stimulus with an established excitatory stimulus during its extinction conditions inhibition to the added stimulus. Not only does the present outcome extend this demonstration of extinctive inhibition to a situational stimulus, but it also provides support for the belief that situational stimuli normally become inhibitory during the course of simple extinction (cf. Rescorla, in press).

With respect to the second issue, the present outcome may bear on Overton's (1972) suggestion that state-dependent learning plays a role in the phenomenon of drug dependence. Specifically, he suggests that, "alcoholics may drink to obtain access to behavioral patterns or emotional reactions which have become conditioned to the presence of alcohol, rather than to obtain any of the intrinsic effects of alcohol" (p. 212). It seems quite possible that alcohol's acquiring of inhibitory properties would increase its value as a positive reinforcer (cf. LoLordo, 1969), and thus may represent an instance of the type of conditioning to which Overton refers.

\section{REFERENCE NOTE}

1. Cunningham, C. L. Escape from fear under alcohol. Manuscript submitted for publication, 1978.

\section{REFERENCES}

AMit, Z., \& BAUM, M. Comment on the increased resistanceto-extinction of an avoidance response induced by certain drugs. Psychological Reports, 1970, 27, 310.

AMIT, Z., Ziskind, D., \& BAuM, M. Drug effects and avoidance extinction in rats: A test of the drug novelty hypothesis using ethanol. Animal Learning \& Behavior, 1973, 1, 41-43.

BARRY, H., III, WAGNer, A. R., \& Miller, N. E. Effects of alcohol and amobarbital on performance inhibited by experimental extinction. Journal of Comparative and Physiological Psychology, 1962, 55, 464-468.

BAUM, M. Paradoxical effect of alcohol on the resistance to extinction of an avoidance response in rats. Journal of Comparative and Physiological Psychology, 1969, 69, 238-240.

BAUM, M. Effect of alcohol on the acquisition and resistanceto-extinction of avoidance responses in rats. Psychological Reports, 1970, 26. 759-765.

Baum, M. Effect of alcohol on the resistance-to-extinction of an avoidance response: Replication in mice. Physiology \& Behavior, 1971, 6, 307-309.

Blackman, D. Conditioned suppression and the effects of classical conditioning on operant behavior. In W. K. Honig \& J. E. R. Staddon (Eds.), Handbook of operant behavior. Englewood Cliffs, N.J: Prentice-Hall, 1977.

Cappell, H., \& Herman, P. C. Alcohol and tension reduction: A review. Quarterly Journal of Studies on Alcohol, 1972, 33, 33-64.

Cicala, G. A., \& Hartley, D. L. Drugs and the learning and performance of fear. Journal of Comparative and Physiological Psychology, 1967, 64, 175.178.

DEUTSCH, J. A., \& HARDY, W. T. Ethanol tolerance in the rat measured by the untasted intake of alcohol. Behavioral Biology, 1976, 17, 379-389. 
Dickerson, L. L., \& Ferraro, D. P. Effects of alcohol on specitic and environmental fear. Psychological Reports, 1976. 39. 1335-1342.

DiGiusso, E. L., \& Bond. N. Enhancement of pseudoconditioning and retardation of escape by low doses of ethanol. Pharmacology, Biochemistry. \& Behavior, 1977, 6, 175-177.

Goldman, P. S., \& Docter, R. F. Facilitation of barpressing and "suppression" of conditioned suppression in cats as a function of alcohol. Psychopharmacologia (Berl.), 1966, 9, 64-72.

LAUENER, H. Conditioned suppression in rats and the effect of pharmacological agents thereon. Psychopharmacologia, 1963, 4, 311-325.

LeBlanc, A. E., \& Cappell, H. D. Historical antecedents as determinants of tolerance to and dependence upon psychoactive drugs. In H. D. Cappell \& A. E. LeBlanc (Eds.), Biological and behavioural approaches to drug dependence. Toronto: Addiction Research Foundation, 1975.

LoLoRDo, V. M. Positive conditioned reinforcement from aversive situations. Psychological Bulletin, 1969, 72, 193-203.
Matchett, J. A., \& Erickson, C. K. Alteration of ethanolinduced changes in locomotor activity by adrenergic blockers in mice. Psychopharmacology, 1977, 52, 201.206.

Overton. D. A. State-dependent learning produced by alcohol and its relevance to alcoholism. In B. Kissin \& H. Begleiter (Eds.), The biology of alcoholism (Vol. 2). New York: Plenum Press, 1972.

Rescorla, R. A. Pavlovian conditioned inhibition. Psychological Bulletin, 1969, 72, 77.94.

Rescorla, R. A. Conditioned inhibition and extinction. In R. A. Boakes \& A. Dickinson (Eds.), Mechanisms of learning and motivation. Hillsdale, N.J: Erlbaum, in press.

Skurdal, A., Eckardt, M. J., \& Brown, J. S. The effects of alcohol on escape learning and on regular and punished extinction in a self-punitive situation with rats. Physiological Psychology, 1975, 3, 29-34.

(Received for publication March 31, 1978; revision accepted July 10,1978 .) 